\title{
Zementaugmentation am proximalen Humerus
}

Michael Plecko

\section{Einleitung}

Die Frakturen des Oberarmkopfs bzw. des proximalen Humerus zählen neben den distalen Radiusfrakturen und den Frakturen des proximalen Femurs zu den häufigsten Frakturen des geriatrischen Patienten. Ihre Inzidenz wird in europäischen Ländern mit ca. 298 Frakturen auf 100000 Einwohner/Jahr angegeben [1]. Frauen sind häufiger betroffen als Männer, und durch die demografische Entwicklung der Bevölkerung ist mit einem weiteren Ansteigen der Anzahl proximaler Humerusfrakturen in unseren Versorgungseinrichtungen zu rechnen. Die Mehrheit dieser Frakturen ist Folge niederenergetischer Traumen, wie einem Sturz beim Gehen im häuslichen Umfeld. Osteoporose reduziert durch die Verminderung der Knochendichte und die typischen Veränderungen der Mikrostruktur die mechanische Kompetenz des Knochens ganz beträchtlich und erhöht dadurch wesentlich die Wahrscheinlichkeit, eine Fraktur zu erleiden [2]. Das Frakturrisiko kann als „Factor of Risk“ angegeben werden, der sich aus dem Quotienten der einwirkenden Kraft (Belastung) dividiert durch die Belastung, bei der der Knochen versagt, errechnet. Ist dieser Wert größer oder gleich 1 muss in hohem Maße mit einer Fraktur gerechnet werden [3].

In der Versorgung proximaler Humerusfrakturen findet heute neben der konservativen Therapie eine Vielzahl verschiedener Behandlungskonzepte Anwendung. Neben minimalinvasiven operativen Stabilisierungstechniken mit perkutan eingebrachten Kirschner-Drähten [4] oder neu entwickelten Implantaten wie dem Humerusblock NG [5] hat in den letzten Jahren die Stabilisierung mit winkelstabilen Plattenimplantaten und winkelstabilen intramedullären Nägeln breite Anwendung gefunden [6-8]. Durch immer bessere Implantate und optimierte Operationstechnik ist die klassische Frakturprothese deutlich in den Hintergrund gerückt [9]. Die Angaben in der Literatur zu Komplikationsraten und klinischen Ergebnissen bez. Osteosynthese vs. Hemiprothese sind jedoch kontrovers $[10,11]$. Obwohl mit den kopferhaltenden Operationsverfahren in einem beachtlichen Ausmaß gute Ergebnisse erzielt werden können, ist doch evident, dass diese bei Patienten mit Osteoporose trotz der verbesserten Implantate und einer verbesserten Operationstechnik nach wie vor mit einer hohen Komplikationsrate be- haftet sind [12-15]. Hierbei stehen die sekundäre Schraubenperforation, der Repositionsverlust mit Varusfehlstellung und die Oberarmkopfnekrose an erster Stelle [16]. Als Risikofaktoren für ein derartiges Implantatversagen konnte das hohe Alter der Patienten, die reduzierte lokale Knochendichte bei Osteoporose, eine ungenügende Reposition der Fraktur und eine fehlende mediale Abstützung am Calcar herausgearbeitet werden [17-19].

\begin{abstract}
Merke
Osteosynthesen weisen bei osteoporotischen proximalen Humerusfrakturen nach wie vor eine hohe Komplikationsrate auf, wobei der schlechte Halt der Schrauben im Oberarmkopf häufig zu einem sekundären Repositionsverlust und einem Cut-out der Schrauben führt. Die verminderte mechanische Kompetenz des osteoporotischen Knochens sowie eine fehlende mediale Abstützung, eine primär ungenügende Reposition der Fraktur und das hohe Alter der Patienten sind die wichtigsten Risikofaktoren für ein Implantatversagen.
\end{abstract}

Die Verwendung rigider Implantate im osteoporotischen Knochen eines Oberarmkopfs mit stark beeinträchtigter mechanischer Kompetenz führt zu einer Überlastung des Knochen-Implantat-Interfaces und scheint für die hohen Cut-out-Raten verantwortlich zu sein [20]. Die Knochendichte und die Architektur der Knochenbälkchen sind innerhalb eines Knochens heterogen verteilt und diese Heterogenität verändert sich mit dem Alter [21]. Diese Verteilung hat einen erheblichen Einfluss auf die biomechanischen Eigenschaften des Knochens. Ebenso ist die Mikrostruktur der Spongiosa in den verschiedenen Sektoren eines Oberarmkopfs unterschiedlich. Die Dicke und das Volumen der Trabekel und damit die mechanische Kompetenz sind am größten im kranialen, medialen und dorsalen Anteil des Humeruskopfs und am geringsten im anterior-superioren Anteil [22,23]. Die Ausrisskräfte einer Schraube sind direkt korreliert mit der lokalen trabekulären Knochendichte [24]. Derzeit wird eine lokale Knochendichte von $95 \mathrm{mg} / \mathrm{cm}^{3}$ als Grenzwert angesehen, unter welchem eine hohe Gefahr für ein mechanisches Implantatversagen durch Versagen des KnochenImplantat-Interfaces besteht [25]. Aus diesem Grund scheint es wesentlich zu sein, sich bei der Behandlung von proximalen Humerusfrakturen beim alten Patienten präoperativ durch exakte Messung einen genauen Über- 

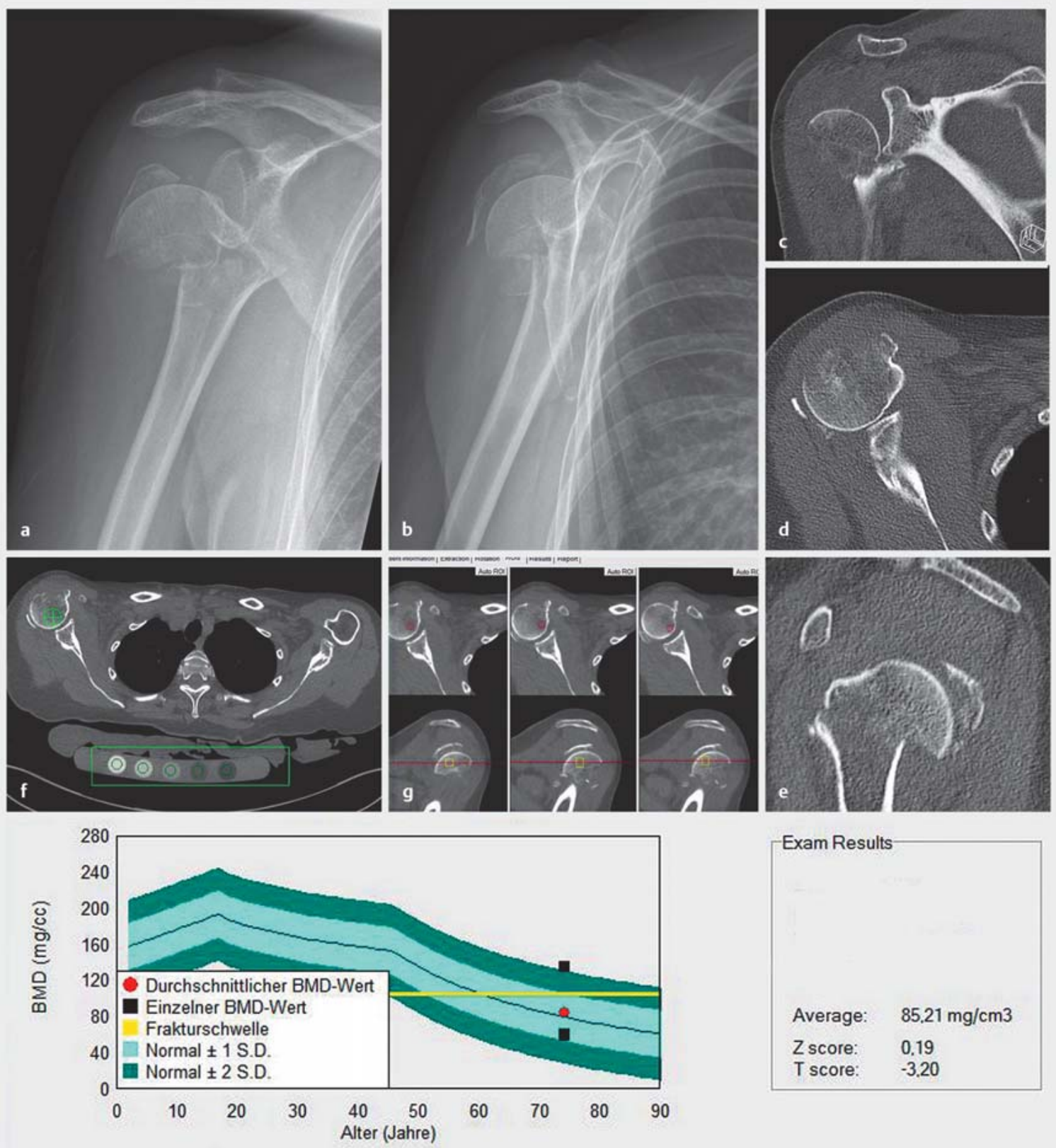

h

BMD Wert des Patienten verglichen mit alters- und geschlechtsbezogenen Kontrolldaten (UCSF)

- Abb. 1 Knochendichtemessung mittels QCT anhand einer 4-Teile-Fraktur des proximalen Humerus (AOT/OTA 11-C2). a, b Nativröntgen. c-e Ausgewählte CT-Schichten in allen 3 Ebenen. $\mathbf{f}$ Referenzphantom. $\mathbf{g}$ Definition einzelner Messzonen. $\mathbf{h}$ Grafische Darstellung des Messergebnisses und des T-Wertes.

blick über die lokale Knochendichte in den einzelnen Sektoren des Oberarmkopfs zu verschaffen. Dies ist am besten mittels QCT-Messung (QCT: quantitative Computertomografie) in den einzelnen Zielgebieten des Oberarmkopfs unter Verwendung eines entsprechenden Phantoms möglich $[26,27]$ ( $\triangleright$ Abb. 1). Auch mechanische Messmethoden (Densiprobe ${ }^{\circledR}$ ) direkt durch die Schraubenlöcher der Platte werden teilweise hierzu verwendet [28], sind aber am Markt noch nicht allgemein zugänglich.

Bei der Osteosynthese proximaler Humerusfrakturen muss durch möglichst exakte, aber dennoch schonende Repositionsmanöver eine hohe intrinsische Stabilität durch kortikale Abstützung angestrebt werden [18,29, 
30]. Zusätzlich sollten die Schrauben der verwendeten Implantate in jenen Bereichen platziert werden, welche die beste Knochenqualität aufweisen. Hier ist durch die vorgegebenen Plattenlöcher meist nur ein beschränkter Spielraum gegeben. Um die Gefahr eines Nachsinkens der Fraktur mit Repositionsverlust und ein SchraubenCut-out zu verringern, wurden in den letzten Jahren verschiedene Verfahren propagiert. Durch Auffüllen des nach der Reposition entstehenden metaphysären Hohlraums mit Kalziumsulfat- bzw. Kalziumphosphatzement [31,32] oder durch eine pilzartig geformte Spongiosaplombe [33] aus der Knochenbank kann ein sekundäres Nachsinken des Kopffragments deutlich vermindert werden. Bei fehlender medialer Abstützung durch einen Defekt oder eine Trümmerzone am Calcar kann ein varisches Abkippen des Oberarmkopfs durch 2 winkelstabile Calcarschrauben sowie durch Einbringen eines kortikospongiösen Knochenblocks [34] oder eines ca. 5 cm langen Fibulaspans verhindert werden [35-37]. Zur Entlastung des Knochen-Implantat-Interfaces wurden auch dynamische Verriegelungsschrauben eingesetzt, die durch ihr elastisches Verhalten und ihr abgerundetes Schraubenende eine deutlich geringere Rate an sekundären Schraubenperforationen zeigten [38].

Um die Qualität des Knochen-Implantat-Interfaces zu verbessern, wurden, motiviert durch die positiven Erfahrungen mit zementaugmentierten Hüftimplantaten, kanülierte Verriegelungsschrauben für den proximalen Humerus entwickelt, die im distalen Anteil der Schraube mehrere Perforationen zum Einbringen von Knochenzement aufweisen. Durch eine passende Einspritzvorrichtung ist es möglich, durch die Schraube hindurch hochviskösen PMMA-Knochenzement (Traumacem $\mathrm{V}+{ }^{\circledR}$ ) um die Schraubenspitze einzubringen, nachdem die Schraube gesetzt wurde. Die empfohlene Zementmenge be-

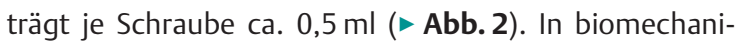
schen Untersuchungen konnte nachgewiesen werden, dass die Zementaugmentation der Schrauben mit eingespritztem PMMA-Zement zu einer signifikant besseren Fixierung der Schraube im Knochen führt [39]. Dadurch konnte eine wesentlich höhere Anzahl von Belastungszyklen ohne Implantatversagen erzielt werden. Die Zementaugmentation der Verriegelungsschrauben ist also in der Lage, die Schwächung des Knochen-Implantat-Interfaces bei osteoporotischen, proximalen Humerusfrakturen zu kompensieren. Dieser positive Effekt ist aber nur bei schlechter Knochenqualität relevant. Weiterhin konnte in einer biomechanischen Studie bei Bewegung unter Simulation aktiver Muskelkräfte klar gezeigt werden, dass der stabilisierende Effekt der Schraubenaugmentation hauptsächlich bei Osteoporose und instabiler Fraktursituation zum Tragen kommt [40]. Durch die Zementaugmentation der Schrauben kommt es zu keiner Veränderung der Implantatsteifigkeit, wohl aber zu einer signifikant geringeren Bewegung am Knochen-Implantat-Interface [41].

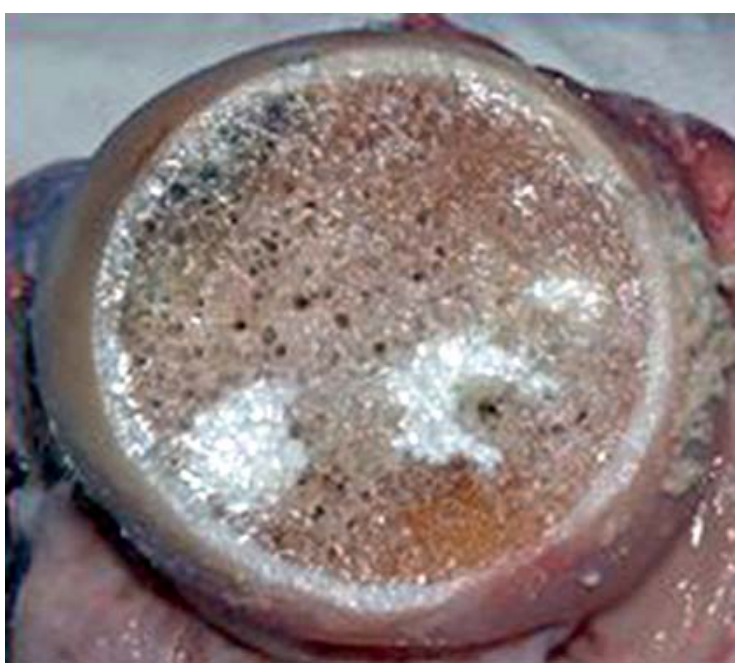

- Abb. 2 Querschnitt eines Humeruskopfs (humanes Leichenpräparat) nach Schraubenaugmentation mit $0,5 \mathrm{ml}$ PMMA-Zement pro Schraube.

\section{Merke}

Die In-situ-Augmentation der Kopfverriegelungsschrauben am proximalen Humerus führt zu einem wesentlich verbesserten Halt der Schrauben im osteoporotischen Knochen und dadurch zu einem viel späteren Versagen des Implantats.

\section{Operationstechnik der Schrauben- augmentation am proximalen Humerus}

Die In-situ-Schraubenaugmentation mit Knochenzement am proximalen Humerus ist ein Add-on-Verfahren. Daher hat diese Technik keinen Einfluss auf Lagerung, Abdeckung oder operativen Zugang. Sie kann sowohl bei der offenen Technik der winkelstabilen Plattenosteosynthese über einen deltoideopektoralen Zugang wie auch bei einer minimalinvasiven Plattenosteosynthese (MIPO) über einen anterolateralen Delta-Split-Zugang angewandt werden. Die Reposition der proximalen Humerusfraktur erfolgt in üblicher Weise durch vorwiegend indirekte Repositionsmanöver, wobei wir im eigenen Vorgehen immer mit dem Legen von Haltefäden an der Rotatorenmanschette (Supraspinatus, Infraspinatus und Subscapularis) beginnen. Die Periostbrücken zwischen den Fragmenten werden belassen, um die Durchblutung der Fragmente nicht weiter zu gefährden. Nach möglichst schonender anatomischer Reposition und, wenn möglich, Wiederherstellung der medialen Abstützung wird die Fraktur zuerst mit Kirschner-Drähten fixiert und anschließend eine winkelstabile Platte $\left(\mathrm{PHILOS}^{\circledR}\right)$ angelegt. Die Drähte dürfen die Gelenkfläche nicht perforieren. Häufig erfolgt auch die Reposition der subkapitalen Frakturkomponente an die Platte durch Heranziehen des Schaftes mit einer Repositionsschraube im ovalen Plattenloch. Bei korrekter 


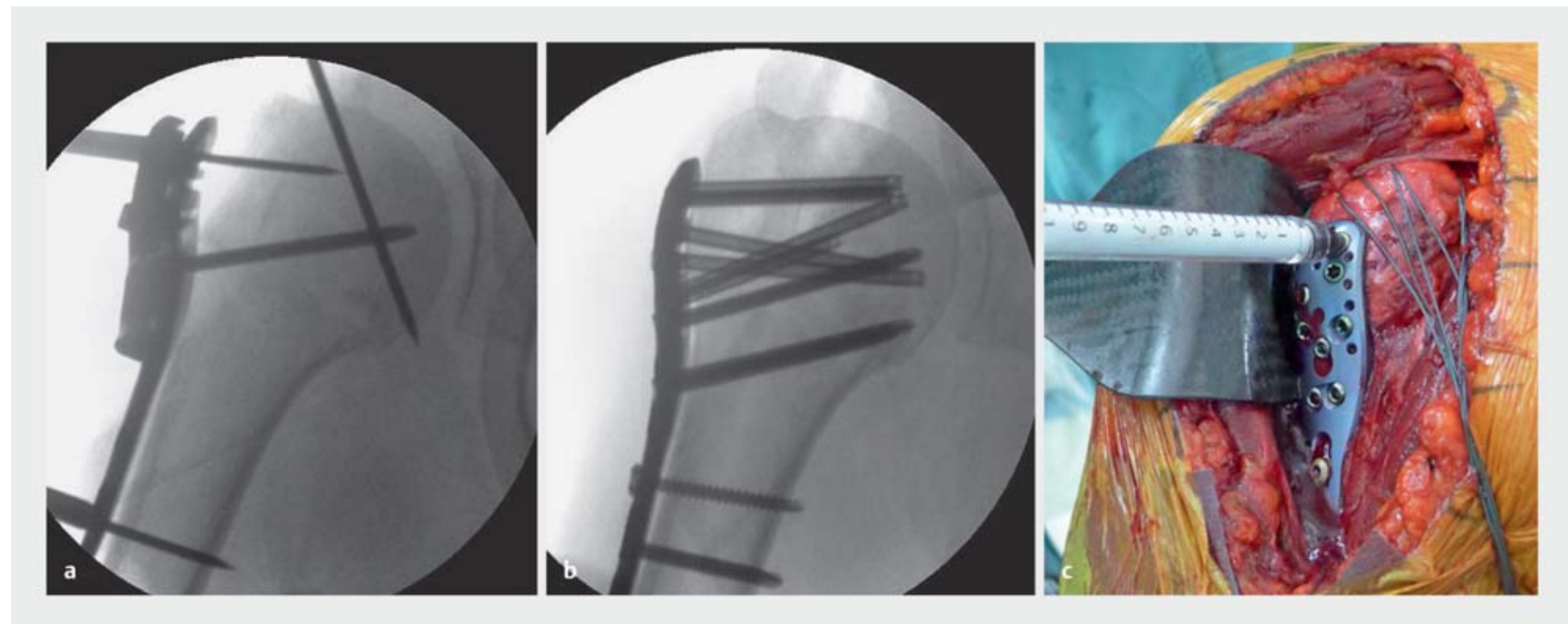

- Abb. 3 Operationstechnik am Beispiel einer 3-Teile-Fraktur des proximalen Humerus (AOT/OTA 11-C2). a Schonende Reposition und temporäre Stabilisierung mit Kirschner-Drähten. b PHILOS ${ }^{\circledR}$-Platte mit kanülierten Kopfverriegelungsschrauben. c Operativer Situs mit angesetzter Spritze mit Knochenzement zur Augmentation der kranialen Schraube.

Stellung werden nun, je nach Frakturform, die einzelnen Plattenlöcher mit winkelstabilen Schrauben besetzt. Ihre Verteilung richtet sich nach der Fraktursituation und der Knochenqualität. Beim Vorbohren muss darauf geachtet werden, nicht in das Gelenk zu perforieren, da dies eine spätere Augmentation unmöglich macht.

Bei Osteoporose verwenden wir am Oberarmkopf häufig kanülierte Kopfverriegelungsschrauben. In Abhängigkeit von der Knochenqualität können diese Schrauben zusätzlich augmentiert werden ( $\triangleright$ Abb. 3). Welche Schrauben mit Knochenzement augmentiert werden, richtet sich im eigenen Vorgehen nach der lokalen Knochenqualität. Hierzu ist eine präoperative Messung mittels QCT in den einzelnen Sektoren des Oberarmkopfs der gesunden Seite hilfreich. Sehr häufig sind es die Schrauben im kranialen ventralen Bereich des Kopfes sowie die zentrale Schraube. Üblicherweise sind es 4 Schrauben, bei denen Knochenzement eingespritzt wird, gelegentlich auch mehr. Die Menge sollte $0,5 \mathrm{ml}$ Zement pro Schraube nicht wesentlich überschreiten, um biologische Nebenwirkungen zu vermeiden. Nach den verfügbaren biomechanischen Daten ist diese Menge zur Stabilisierung absolut ausreichend. In Simulationsmodellen sind die verschiedenen Augmentationsmuster auf ihre biomechanische Kompetenz untersucht worden. Daraus ergibt sich, dass eine Augmentation von mindestens 4 Schrauben, möglichst breit im Humeruskopf verteilt, günstig wäre [42]. In einer biomechanischen Studie konnte gezeigt werden, dass durch Augmentation der 4 proximalen Schrauben einer PHILOS-Platte eine deutlich verbesserte Stabilität mit annähernd 54\% mehr tolerierten Belastungszyklen erzielt werden konnte [39]. Durch exakte Messung der Knochenqualität in den einzelnen Zielgebie-

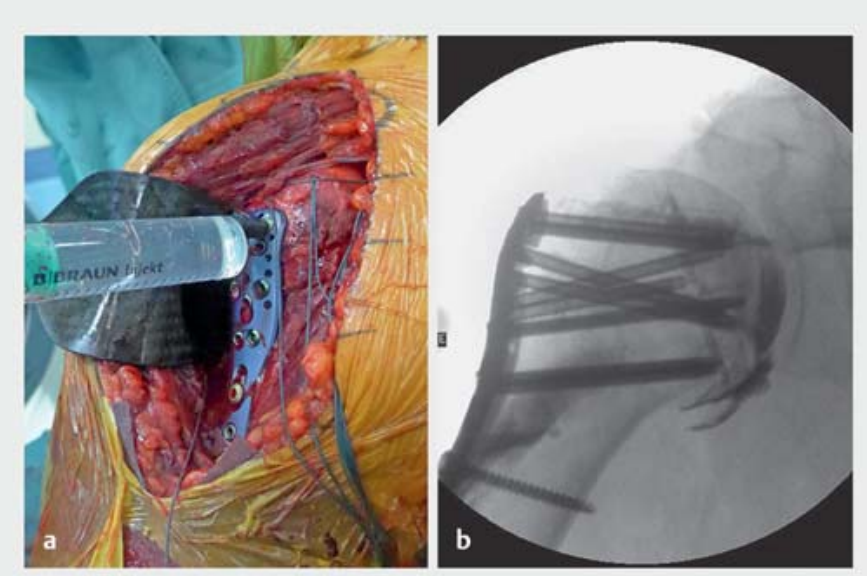

Abb. 4 Prüfen auf Vorliegen einer Leckage vor dem Durchführen der Augmentation. a Operativer Situs: Angesetzte Injektionsspritze mit verdünntem Röntgenkontrastmittel. b Bildwandlerbild mit deutlichem Austritt von Kontrastmittel in das Schultergelenk. In diesem Fall verbietet sich das Einspritzen von Zement durch die geprüfte Schraube.

ten der Kopfverriegelungsschrauben konnte durch Augmentation ausschließlich der beiden Schrauben, die in den schlechtesten Knochenarealen zu liegen kommen (meist die beiden Schrauben im ventralen Kopfanteil) eine annähernd gleich große Verbesserung der Anzahl an Belastungszyklen (50\%) erreicht werden [43].

Wichtig ist es, vor dem Einbringen des Knochenzements durch Einspritzen von Röntgenkontrastmittel bei jeder Schraube, die augmentiert werden soll, zu prüfen, ob eine Leckage besteht und im Bildwandler ein Kontrast- 

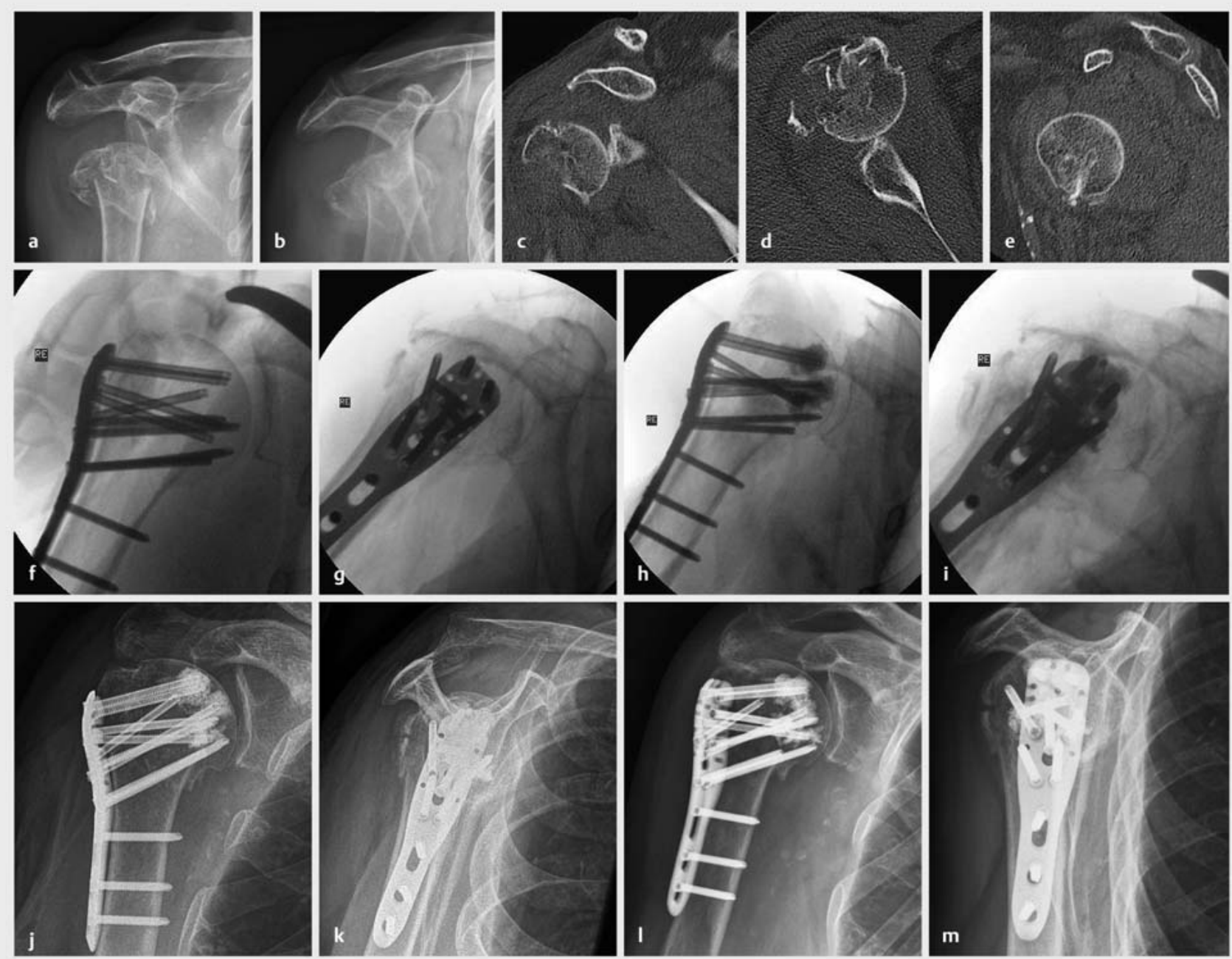

- Abb. 5 71-jährige Patientin. Häuslicher Sturz. Luxationsfraktur proximaler Humerus rechts (AOT/OTA 11-C3) bei beträchtlicher Osteoporose. a, b Nativröntgen. c-e Ausgewählte CT-Schnitte in allen 3 Ebenen. f, g Bildwandlerbilder a.-p. und Outlet nach Reposition und Stabilisierung mit einer PHILOS-Platte. h, i Augmentation von 4 Schrauben mit Knochenzement. j, k Postoperatives Röntgen a.-p. und Outlet View. I, m Röntgen nach 6 Wochen a.-p. und Outlet View - unverändert gute Stellung.

mittelaustritt in das Gelenk zu erkennen ist ( $\bullet$ Abb. 4). In einem solchen Fall darf die besagte Schraube nicht augmentiert werden. Es ist zu empfehlen, das Kontrastmittel so gut als möglich nach Überprüfung jeder Schraube wieder abzusaugen, um die Übersicht zu wahren.

\section{Merke}

Am proximalen Humerus werden die Kopfverriegelungsschrauben, die in stark osteoporotischen Knochenarealen zu liegen kommen, mit einer Menge von ca. 0,5 ml Knochenzement je Schraube augmentiert. Zuvor ist in jedem Fall durch Einspritzen eines Röntgenkontrastmittels eine Leckage in das Gelenk auszuschließen.

Ist eine Schraube zur Augmentation geeignet, wird der Adapter dicht in die Innenbohrung der Schraube einge- setzt und mit einer Injektionsspritze das Innere der Schraube mit Knochenzement befüllt (ca. 0,15 ml). Anschließend werden dann ca. 0,5 ml Knochenzement um die Schraubenspitze platziert. Den Austritt und die korrekte Verteilung des Zements an der Schraubenspitze verfolgen wir üblicherweise mit dem Bildwandler. Abschließend wird der Knochenzement aus dem Inbus der Schraube mit einem Häkchen entfernt. Ein Aushärten des im eigenen Vorgehen verwendeten Knochenzements (Traumacem $\mathrm{V}+{ }^{\circledR}$ ) erfolgt bei Körpertemperatur in ca. 15 Minuten. Es ist unerlässlich, sich vor der Operation über die Eigenschaften (Verarbeitungszeit und Aushärtungszeit) des verwendeten Zements exakt zu informieren. Sollte trotz aller Vorsichtsmaßnahmen dennoch Zement in das Gelenk ausgetreten sein, muss eine Schultergelenksarthroskopie erfolgen, um diese Zementanteile zu entfernen. 


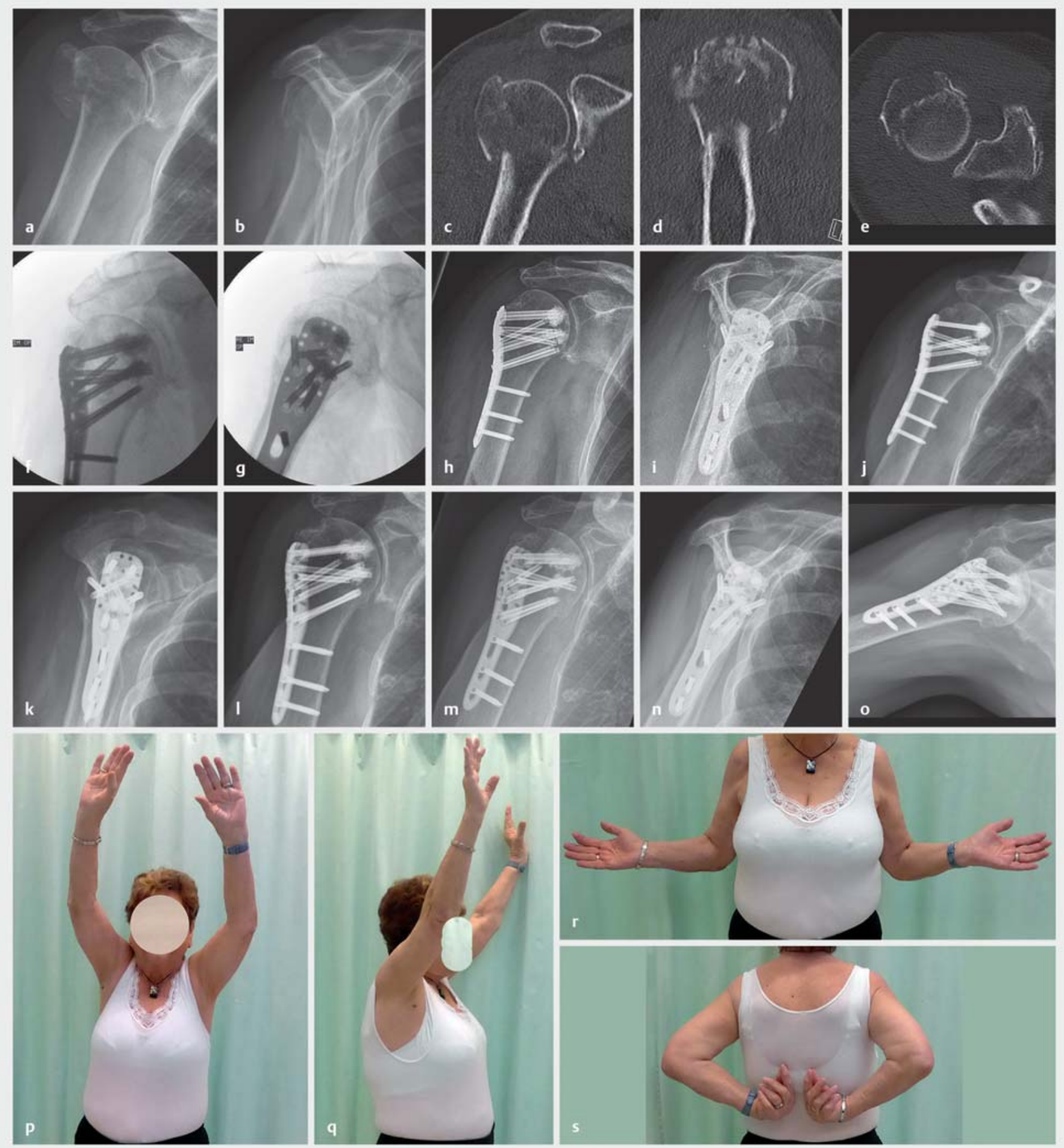

- Abb. 6 75-jährige Pensionistin. Sturz im häuslichen Umfeld. Valgisch impaktierte 4-Teile-Fraktur des rechten proximalen Humerus und ausgeprägte Osteoporose. a, b Primäres Nativröntgen a.-p. und Outlet View. c-e Ausgewählte CT-Schnitte in allen 3 Ebenen. f, g Bildwandlerbilder a.-p. und Outlet View nach Reposition und Stabilisierung mit einer PHILOS-Platte und zusätzlicher Schraubenaugmentation. h, i Postoperatives Röntgen a.-p. und Outlet View. j, k Röntgenkontrolle nach 3 Wochen a.-p. und Outlet View - unverändert gute Stellung. I-o Nativröntgen a.-p., schräg, Outlet View und axillär 2 Jahre postoperativ - Fraktur in unverändert guter Stellung knöchern geheilt. p-s Funktion nach 2 Jahren (Abduktion, Flexion, Außenrotation, Innenrotation); die Patientin ist schmerzfrei und hat seitengleiche Kraft.

Anschließend werden die Zuggurtungsfäden durch die Plattenlöcher geführt und geknüpft. Der weitere Operationsablauf entspricht dem üblichen Vorgehen $(\bullet$ Abb. 5 und 6).
Die beim Aushärten des Zements entstehende Wärme darf nicht zur Knochen- oder Knorpelnekrose führen. Für die dargestellte Schraubenaugmentationstechnik am proximalen Humerus mit einer PHILOS-Platte, kanülier- 
ten Schrauben und Traumacem V+ konnte dies in einer aufwendigen Studie ausgeschlossen werden [44]. Die gemessenen Werte erreichten maximal ca. $43^{\circ} \mathrm{C}$ für $45 \mathrm{Se}$ kunden in einem „worst case scenario“ mit einem geringen Abstand der Schraubenspitze von 5 mm zur Knorpelfläche.

\section{Ergebnisse}

Bei der In-situ-Augmentation von winkelstabilen Schrauben in Verbindung mit einer Plattenosteosynthese am proximalen Humerus handelt es sich um ein sehr aktuelles, erst kürzlich entwickeltes Verfahren. Daher sind zwar ausreichend Grundlagendaten vorhanden, hochwertige klinische Daten fehlen aber noch. In der eigenen ersten Fallserie (20 Patienten) konnte keine verfahrensspezifische Komplikation festgestellt werden. In einem Fall ist es bei primär ungenügender Reposition bei einer sehr komplexen 4-Segment-Fraktur mit Trümmerzone zu einem Repositionsverlust gekommen, und in 2 Fällen ist nach anfänglich gutem Verlauf bei primär avitalem Kopfsegment nach mehr als 12 Monaten doch noch eine partielle Kopfnekrose aufgetreten. Bei beiden Patientinnen stehen die Schraubenenden nicht über die Kopfkontur vor, und die Patientinnen sind mit dem Ergebnis zufrieden, obwohl eine eingeschränkte Beweglichkeit vorliegt. In allen übrigen Fällen ist es zu keinem Repositionsverlust oder Cut-out der Schrauben gekommen.

\section{Schlussfolgerung}

Die Zementaugmentation von Kopfverriegelungsschrauben mit Knochenzement ist bei richtiger Indikation und technisch einwandfreier Durchführung ein sicheres Verfahren, um bei schlechter Knochenqualität den Halt der Implantate im osteoporotischen, spongiösen Knochen des Humeruskopfs zu verbessern. Durch eine bessere Lastverteilung kommt es zu einem Entlasten des Knochen-Schrauben-Interfaces und dadurch zu einem geringeren Schrauben-Cut-out. Dies verhindert ein sekundäres Nachsinken und damit einen Repositionsverlust. Dieser positive Effekt ist umso wirksamer, je schlechter die Knochenqualität ist. Neben anderen erfolgreichen Verfahren zur Augmentation der Osteosynthese bei osteoporotischer proximaler Humerusfraktur wie z.B. durch Verwendung von Allografts, ist die Schraubenaugmentation in Kombination mit einer winkelstabilen Plattenosteosynthese ein geeignetes Verfahren, um auch bei diesen schwierigen Fraktursituationen ein gutes Ergebnis zu erzielen und Komplikationen zu vermeiden.

\section{Interessenkonflikt}

Der Autor gibt an, dass kein Interessenkonflikt besteht.
Über die Autoren

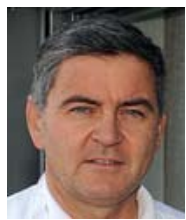

\section{Michael Plecko}

Prim. Dr. med., Ärztlicher Leiter, Unfallkrankenhaus Graz der AUVA, Lehrkrankenhaus der MEDUNI Graz

\section{Korrespondenzadresse}

Prim. Dr. med. Michael Plecko

Ärztlicher Leiter

Unfallkrankenhaus Graz der AUVA

Lehrkrankenhaus der MEDUNI Graz

Göstingerstrasse 24

$8020 \mathrm{Graz}$

Österreich

michael.plecko@auva.at

\section{Literatur}

[1] Kannus P, Palvanen M, Niemi S et al. Rate of proximal humeral fractures in older Finnish women between 1970 and 2007. Bone 2009; 44: 656-559

[2] Reginster JY, Sarlet N, Lecart MP. Fractures in osteoporosis: the challenge for the new millenium. Osteoporos Int 2005; 16 (Suppl. 1): S1-S3

[3] Silva M]. Biomechanics of osteoporotic fractures. Injury 2007; 38 (Suppl. 3): S69-S76

[4] Resch H, Hubner C, Schwaiger R. Minimally invasive reduction and osteosynthesis of articular fractures of the humeral head. Injury 2001; 32 (Suppl. 1: SA25-SA32

[5] Brunner A, Resch H, Babst et al. The Humerusblock NG: a new concept for stabilization of proximal humeral fractures and its biomechanical evaluation. Arch Orthop Trauma Surg 2012; 132: 985-992

[6] Brunner F, Sommer C, Bahrs C et al. Open reduction and internal fixation of proximal humerus fractures using a proximal humeral locking plate: a prospective multicenter analysis. J Orthop Trauma 2009; 23: 163-172

[7] Rothstock S, Plecko M, Kloub M. Biomechanical evaluation of two intramedullary nailing techniques with different locking options in a three-part fracture proximal humerus model. Clin Biomech (Bristol, Avon) 2012; 27: 686-691

[8] Wanzl M, Foehr P, Schreiber U et al. Biomechanical testing to evaluate the cut-through resistance of intramedullary nails for the proximal humerus. Injury 2016; 47 (Suppl. 7): S20-S24

[9] Cvetanovich GL, Chalmers PN, Verma NN et al. Open reduction internal fixation has fewer short-term complications than shoulder arthroplasty for proximal humeral fractures. J Shoulder Elbow Surg 2016; 25: 624-631

[10] Khurana S, Davidovitch RI, Kwon YK et al. Similar function and improved range of motion is achieved following repair of three- and four-part proximal humerus fractures compared with hemiarthroplasty. Bull Hosp Jt Dis 2016; 74: 212-218

[11] Cai M, Tao K, Yang $C$ et al. Internal fixation versus shoulder hemiarthroplasty for displaced 4-part proximal humeral fractures in elderly patients. Orthopedics 2012; 35: e1340-e1346 
[12] Südkamp N, Bayer ], Hepp P et al. Open reduction and internal fixation of proximal humeral fractures with use of the locking proximal humerus plate. Results of a prospective, multicenter, observational study. J Bone Joint Surg Am 2009; 91: 1320-1328

[13] Jost B, Spross C, Grehn H et al. Locking plate fixation of fractures of the proximal humerus: analysis of complications, revision strategies and outcome. J Shoulder Elbow Surg 2013; 22: 542-549

[14] Brorson S, Rasmussen JV, Frich LH et al. Benefits and harms of locking plate osteosynthesis in intraarticular (OTA Type C) fractures of the proximal humerus: a systematic review. Injury 2012; 43: 999-1005

[15] Gönç U, Atabek M, Teker K et al. Minimally invasive plate osteosynthesis with PHILOS plate for proximal humerus fractures. Acta Orthop Traumatol Turc 2017; 51: 17-22

[16] Sproul RC, lyengar JJ, Devcic Z et al. A systematic review of locking plate fixation of proximal humerus fractures. Injury 2011; 42: 408-413

[17] Krappinger D, Bizzotto N, Riedmann S et al. Predicting failure after surgical fixation of proximal humerus fractures. Injury 2011; 42: 1283-1288

[18] Hertel R. Fractures of the proximal humerus in osteoporotic bone. Osteoporos Int 2005; 16 (Suppl. 2): S65-S72

[19] Micic ID, Kim KC, Shin DJ et al. Analysis of early failure of the locking compression plate in osteoporotic proximal humerus fractures. J Orthop Sci 2009; 14: 596-601

[20] Thanasas C, Kontakis G, Angoules A et al. Treatment of proximal humerus fractures with locking plates: a systematic review. J Shoulder Elbow Surg 2009; 18: 837-844

[21] Osterhoff G, Morgan EF, Shefelbine S] et al. Bone mechanical properties and changes with osteoporosis. Injury 2016; 47 (Suppl. 2): S11-S20

[22] Hepp P, Lill H, Bail H et al. Where should implants be anchored in the humeral head? Clin Orthop Relat Res 2003; 415: 139147

[23] Liew ASL, Johnson JA, Patterson SD et al. Effect of screw placement on fixation in the humeral head. J Shoulder Elbow Surg 2000; 9: 423-426

[24] Tingart M], Lehtinen J, Zurakowski D et al. Proximal humeral fractures: regional differences in bone mineral density of the humeral head affect the fixation strength of cancellous screws. J Shoulder Elbow Surg 2006; 15: 620-624

[25] Konstantinidis L, Helwig P, Hirschmüller $A$ et al. When is the stability of a fracture fixation limited by osteoporotic bone? Injury 2016; 47 (Suppl. 2): S27-S32

[26] Krappinger D, Roth T, Gschwentner M et al. Preoperative assessment of the cancellous bone mineral density of the proximal humerus using CT data. Skeletal Radiol 2012; 41: 299304

[27] Brianza S, Röderer $G$, Schiuma D et al. Where do locking screws purchase in the humeral head? Injury 2012; 43: 850855

[28] Röderer G, Brianza S, Schiuma D et al. Mechanical assessment of local bone quality to predict failure of locked plating in a proximal humerus fracture model. Orthopedics 2013; 36: e1134-e1140

[29] Plecko M, Kraus A. Internal fixation of proximal humerus fractures using the locking proximal humerus plate. Oper Orthop Traumatol 2005; 17: 25-50

[30] Konrad G, Bayer J, Hepp P et al. Open reduction and internal fixation of proximal humeral fractures with use of the locking proximal humerus plate. Surgical technique. J Bone Joint Surg Am 2010; 92 (Suppl. 1): S85-S95
[31] Somasundaram K, Huber CP, Babu V et al. Proximal humeral fractures: the role of calcium sulfate augmentation and extended deltoid splitting approach in internal fixation using locking plates. Injury 2013; 44: 481-487

[32] Egol KA, Sugi MT, Ong CC et al. Fracture site augmentation with calcium phosphate cement reduces screw penetration after open reduction-internal fixation of proximal humeral fractures. J Shoulder Elbow Surg 2012; 21: 741-748

[33] Euler SA, Hengg C, Wambacher $M$ et al. Allogenic bone grafting for augmentation in two-part proximal humeral fracture fixation in a high-risk patient population. Arch Orthop Trauma Surg 2015; 135: 79-87

[34] Katthagen JC, Schwarze M, Meyer-Kobbe J et al. Biomechanical effects of calcar screws and bone block augmentation on medial support in locked plating of proximal humeral fractures. Clin Biomech (Bristol, Avon) 2014; 29: 735-741

[35] Osterhoff G, Baumgartner D, Favre P et al. Medial support by fibula bone graft in angular stable plate fixation of proximal humeral fractures: an in vitro study with synthetic bone. J Shoulder Elbow Surg 2011; 20: 740-746

[36] Saltzman BM, Erickson B], Harris JD et al. Fibular strut graft augmentation for open reduction and internal fixation of proximal humerus fractures: a systematic review and the author's preferred surgical technique. Orthop J Sports Med 2016; 4: 19

[37] Hinds RM, Garner MR, Tran WH et al. Geriatric proximal humeral fracture patients show similar clinical outcomes to non-geriatric patients after osteosynthesis with endosteal fibular strut allograft augmentation. J Shoulder Elbow Surg 2015; 24: 889-896

[38] Freude T, Schroeter S, Plecko M et al. Dynamic-locking-screw (DLS) - leads to less secondary screw perforations in proximal humerus fractures. BMC Musculoskelet Disord 2014; 15: 194

[39] Unger S, Erhart S, Kralinger $F$ et al. The effect of in situ augmentation on implant anchorage in proximal humeral head fractures. Injury 2012; 43: 1759-1763

[40] Kathrein S, Kralinger F, Blauth M et al. Biomechanical comparison of an angular stable plate with augmented and non-augmented screws in a newly developed shoulder test bench. Clin Biomech (Bristol, Avon) 2013; 28: 273-277

[41] Schliemann B, Seifert R, Rosslenbroich SB et al. Screw augmentation reduces motion at the bone-implant interface: a biomechanical study of locking plate fixation of proximal humeral fractures. J Shoulder Elbow Surg 2015; 24: 1968-1973

[42] Widmer D, Muench C, Forte M et al. Cement augmentation of a proximal humerus plate for osteoporotic fracture. Numerical analysis of a complex problem. Eur J Trauma Emerg Surg 2014; 40 (Suppl. 1): S11 - 0023

[43] Röderer G, Scola A, Schmölz W et al. Biomechanical in vitro assessment of screw augmentation in locked plating of proximal humerus fractures. Injury 2013; 44: 1327-1332

[44] Blazejak M, Hofmann-Fliri L, Büchler L et al. In vitro temperature evaluation during cement augmentation of proximal humerus plate screw tips. Injury 2013; 44: 1321-1326

Bibliografie

DOI http://dx.doi.org/10.1055/s-0043-102244

OP-JOURNAL 2017; 33: 64-71 @ Georg Thieme Verlag KG Stuttgart · New York ISSN 0178-1715 